\title{
Luminosity geometric reduction factor from colliding bunches with different lengths
}

\author{
S. Verdú-Andrés
}

\section{Collider-Accelerator Department Brookhaven National Laboratory Upton, NY 11973}

\author{
U.S. Department of Energy \\ Office of Science, Office of Nuclear Physics
}

Notice: This document has been authorized by employees of Brookhaven Science Associates, LLC under Contract No. DE-SC0012704 with the U.S. Department of Energy. The United States Government retains a nonexclusive, paid-up, irrevocable, world-wide license to publish or reproduce the published form of this document, or allow others to do so, for United States Government purposes. 


\section{DISCLAIMER}

This report was prepared as an account of work sponsored by an agency of the United States Government. Neither the United States Government nor any agency thereof, nor any of their employees, nor any of their contractors, subcontractors, or their employees, makes any warranty, express or implied, or assumes any legal liability or responsibility for the accuracy, completeness, or any third party's use or the results of such use of any information, apparatus, product, or process disclosed, or represents that its use would not infringe privately owned rights. Reference herein to any specific commercial product, process, or service by trade name, trademark, manufacturer, or otherwise, does not necessarily constitute or imply its endorsement, recommendation, or favoring by the United States Government or any agency thereof or its contractors or subcontractors. The views and opinions of authors expressed herein do not necessarily state or reflect those of the United States Government or any agency thereof. 


\title{
Luminosity geometric reduction factor from colliding bunches with different lengths
}

\author{
S. Verdú-Andrés (sverdu@bnl.gov) \\ Brookhaven National Laboratory, Upton, USA
}

September 29, 2017

\begin{abstract}
In the interaction point of the future electron-Ion collider eRHIC, the electron beam bunches are at least one order of magnitude shorter than the proton beam bunches. With the introduction of a crossing angle, the actual number of collisions resulting from the bunch collision gets reduced. Here we derive the expression for the luminosity geometric reduction factor when the bunches of the two incoming beams are not equal.
\end{abstract}

\section{Introduction}

The electron-Ion collider eRHIC will collide $275 \mathrm{GeV}$ proton beams and $18 \mathrm{GeV}$ electron beams during high energy operations. Electron and proton bunches will have the same transverse dimensions, but different longitudinal dimensions. For the no-cooling, high-divergence lattice, the rms bunch width (horizontal dimension) will be $0.123 \mathrm{~mm}$ and the $\mathrm{rms}$ bunch height (vertical dimension) will be $0.016 \mathrm{~mm}$. The rms length (longitudinal dimension) of the proton bunches will be $70 \mathrm{~mm}$, whereas the rms length of the electron bunches will be $5.8 \mathrm{~mm}$ [1]. The two incoming beams will collide in the horizontal plane with a 22 mrad crossing angle.

The introduction of such crossing angle will lead to a reduction of the luminosity due to the ineffective overlapping of the encountering bunches. The luminosity reduction is a combination of a purely geometric component (quantified by the so-called geometric reduction factor) and the impact of beam-beam effects. The geometric reduction factor depends on the crossing angle and the bunch aspect ratio in the plane of crossing, as the crossing angle increases the effective bunch size in the transverse dimension of the plane of crossing [2]. The aspect ratio for the proton and electron bunches of eRHIC is 569 and 47, respectively [1].

Many publications (for example, Ref. [3, 4, 5, 6]) quote the geometry reduction factor for colliding beams with identical size bunches. In all cases a global Piwinski angle is defined. Such global Piwinski angle comprises the contribution of the two identical colliding bunches. However, the author of this paper could not find the geometry reduction factor for collisions of bunches with different lengths. In this note an individual Piwinski angle is defined for the bunches of each beam and the geometry reduction angle for the collision of bunches with different lengths is provided.

\section{Luminosity of two colliding bunches}

Assume that the two colliding beams bunches with a Gaussian distribution. The luminosity $\mathscr{L}$ resulting from the collision of two Gaussian bunches is given by the following expression [7]:

$$
\mathscr{L}=f_{0} N_{1} N_{2} \frac{\cos (\theta / 2)}{2 \pi} \frac{1}{\sqrt{\left(\sigma_{x, 1}^{2}+\sigma_{x, 2}^{2}\right)}} \times \frac{1}{\sqrt{\left(\sigma_{y, 1}^{2}+\sigma_{y, 2}^{2}\right) \cos ^{2}(\theta / 2)+\left(\sigma_{z, 1}^{2}+\sigma_{z, 2}^{2}\right) \sin ^{2}(\theta / 2)}}
$$

where $f_{0}$ is the revolution frequency, $N_{i}$ is the number of particles in bunch number $i, \theta$ is the crossing angle and $\sigma_{j, i}$ is the size of bunch number $i$ in a given direction $j=x, y, z$.

The luminosity is maximal in case of head-on collisions $(\theta=0)$ : 


$$
\mathscr{L}_{H O}=f_{0} N_{1} N_{2} \frac{1}{2 \pi} \frac{1}{\sqrt{\left(\sigma_{x, 1}^{2}+\sigma_{x, 2}^{2}\right)}} \frac{1}{\sqrt{\left(\sigma_{y, 1}^{2}+\sigma_{y, 2}^{2}\right)}}
$$

The luminosity reduction factor $\mathscr{F}$ due to the introduction of a crossing angle is just the ratio of the luminosity for a given crossing angle over the luminosity for head-on collisions, defined as:

$$
\mathscr{F}=\frac{\mathscr{L}}{\mathscr{L}_{H O}}=\frac{1}{\sqrt{1+\left(\frac{\sigma_{z, 1}^{2}+\sigma_{z, 2}^{2}}{\sigma_{y, 1}^{2}+\sigma_{y, 2}^{2}}\right) \tan ^{2}(\theta / 2)}}
$$

The crossing angle is typically very small, $\theta \ll 1$. For example, $\theta$ is only $590 \mu \mathrm{m}$ for HL-LHC and 22 mrad for eRHIC. Thus, one can use the approximation: $\tan (\theta / 2) \approx(\theta / 2)$. In addition we also assume that the bunches of the two incoming beams have the same transverse dimensions (like eRHIC), so one can simplify: $\sigma_{j, 1}=\sigma_{j, 2}=\sigma_{y}$. With all these assumptions, the reduction factor takes the form:

$$
\mathscr{F} \simeq \frac{1}{\sqrt{1+\frac{\sigma_{z, 1}^{2}}{2 \sigma_{y}^{2}}\left(\frac{\theta}{2}\right)^{2}+\frac{\sigma_{z, 2}^{2}}{2 \sigma_{y}^{2}}\left(\frac{\theta}{2}\right)^{2}}}
$$

The Piwinski angle $\Phi_{i}$ for a given bunch is the aspect ratio of the bunch in the crossing plane times the crossing angle held by the two incoming beams:

$$
\Phi_{i}=\frac{\sigma_{z, i}}{\sigma_{y}}\left(\frac{\theta}{2}\right)
$$

The expression of the luminosity reduction factor $\mathscr{F}$ is simplified when written as a function of the Piwinski angle as follows:

$$
\mathscr{F}=\frac{1}{\sqrt{1+\frac{1}{2} \Phi_{1}^{2}+\frac{1}{2} \Phi_{2}^{2}}}
$$

If the bunches of one beam are much longer than the bunches of the other beam $\left(\sigma_{z, 1} \gg \sigma_{z, 2}\right)$, then the Piwinski angle of the beam with longer bunches will determine the value of the luminosity reduction factor: $\mathscr{F} \approx\left(1+\frac{1}{2} \Phi_{1}^{2}\right)^{-1 / 2}$. This is the case for the future electron-ion collider eRHIC [1]. When the bunches of the two colliding beams have the same length, $\sigma_{z, 1}=\sigma_{z, 2}=\sigma_{z}$, then Eq. 7 becomes the familiar expression found in other literature:

$$
\mathscr{F}=\frac{1}{\sqrt{1+\Phi^{2}}}
$$

where the Piwinski angle $\Phi$ is simply:

$$
\Phi=\frac{\sigma_{z}}{\sigma_{y}}\left(\frac{\theta}{2}\right)
$$

The Piwinski angle $\Phi$ is generally a small number, lower than unity $(\Phi \ll 1)$. In consequence, the luminosity reduction factor $\mathscr{F}$ can be approximated using the Taylor expansion to: $\mathscr{F} \approx 1-\Phi^{2} / 2$.

\section{Future colliders: luminosity reduction due to crossing angle}

The nominal collision parameters for HL-LHC and eRHIC are summarized in Table 1 The large luminosity reduction factor in HL-LHC (30\%) motivated the development of crab crossing system to reestablish headon collisions [8]. Crab crossing will also be implemented in eRHIC to reach a nominal luminosity of $10^{34}$ $\mathrm{cm}^{-2} \mathrm{~s}^{-1}[9]$.

\section{Acknowledgements}

I would like to thank Yue Hao for suggesting me write these expressions down and also Ilan Ben-Zvi for encouraging me to publish this personal note. This work was supported by the US Department of Energy via Brookhaven Science Associates LLC under contract no. DE-SC0012704. 
Table 1: Nominal collision parameters in future particle colliders

\begin{tabular}{lcccl}
\hline & & $\begin{array}{c}\text { HL-LHC [10] } \\
\text { (proton, proton) }\end{array}$ & $\begin{array}{c}\text { eRHIC [1] } \\
\text { (electron, proton) }\end{array}$ & \\
\hline Crossing angle & $\theta$ & 0.59 & 22 & $\mathrm{mrad}$ \\
Bunch length & $\sigma_{z}$ & $(75.5,75.5)$ & $(5.8,70)$ & $\mathrm{mm}$ \\
Bunch width & $\sigma_{y}$ & $(0.007,0.007)$ & $(0.123,0.123)$ & $\mathrm{mm}$ \\
Piwinski angle & $\Phi_{i}$ & $(3.18,3.18)$ & $(0.52,6.26)$ & $\mathrm{rad}$ \\
Reduction factor & $\mathscr{F}$ & 0.30 & 0.22 & $\%$ \\
\hline
\end{tabular}

\section{References}

[1] V. Ptitsyn, eRHIC Parameters v3, unpublished (2017).

[2] F. Zimmerman, "Collider beam physics", CERN-ACC-2014-0284 (2014). Accessed online: https:// inspirehep.net/record/1343874/files/CERN-ACC-2014-0284.pdf

[3] A. Chao et al., Ed. "Handbook of accelerator physics and engineering", World Scientific (2013).

[4] W. Herr and B. Muratori, "Concept of luminosity", in Proc. CERN Accelerator School, Zeuthen 2003, CERN-2006-002 (2006). Accessed online: https://cds.cern.ch/record/941318/files/p361. pdf

[5] M. Syphers, "Some notes on Luminosity Calculations", Fermilab note, Beams-doc-1348 (2007). Accessed online: http://home.fnal.gov/ syphers/Accelerators/tevPapers/LumiCalc. $\mathrm{pdf}$

[6] P. Raimondi, D. Shatilov and M. Zobov, "Beam-beam issues for colliding schemes with large Piwinski angle and crabbed waist", in Proc. of PAC'07, Albuquerque, New Mexico, TUPAN047 (2007). Accessed online: https://arxiv.org/pdf/physics/0702033.pdf

[7] H. Damerau, "Creation and storage of long and flat bunches in the LHC", Technische Universität, Darmstadt, Chapter 4, p. (2005). Accessed online: http://elib.tu-darmstadt.de/diss/ 000612

[8] R. Calaga, "Crab cavities for the LHC upgrade", in Proc. of Chamonix 2012 workshop on the LHC performance, Chamonix (2012).

[9] C. Montag et al., "Overview of the eRHIC ring-ring design", in Proc. of IPAC'17, Copenhagen, WEPIK049 (2017). Accessed online: http://accelconf.web.cern.ch/AccelConf/ipac2017/ papers/wepik049.pdf

[10] I. Bejar et al., "The HL-LHC Machine" in The high luminosity large hadron collider, O. Bruning and L. Rossi, Ed. CERN, Geneva, Switzerland: World Scientific, 2015, pp. 31-44. Accessed online: https: //cds.cern.ch/record/2130741/files/The $\% 20 H L-L H C: 20 M a c h i n e . p d f$ 